\title{
Determinación del sexo antes del nacimiento
}

(Artículo reproduciđo del "Día Médico", de Buenos Aires, del 12 de julio de 1956).

Dos biólogos y un ginecólogo israelíes, al examinar el fluído amniótico extraído de la matriz de 20 mujeres encinta, durante el noveno mes de gestación, descubrieron que contenía células que, al analizarlas, proporcionaban un diagnóstico correcto del sexo de la criatura próxima a nacer. En un caso, en el sexto mes cie embarazo, como la paciente sufría de hidramnios, tuvo que extraerse el exceso de fluído, y el mismo análisis resultó igualmente correcto. En resumen, habian encontrado la respuesta antes del nacimiento de la criatura sobre si sería niño o niña.

El doctor David M. Serr, ginecólogo de la Organización Médica de Hadassah, en Jerusalén, en compañía del doctor Leo Sachs y la doctora Matilde Danon, de la Sección de Biología Experimental del Instituto Científico Weizmann, publicaron su primer informe sobre el descubrimiento en el "Boletín del Consejo de Investigaciones de Israel" (volumen 5-b, páginas 137-138. 1955), bajo el título "La diagnosis del sexo antes del nacimiento, utilizando células del fluído amniótico”.

En su introducción al informe dice: "Entre los seres humanos, lo mismo que entre la mayoría de los mamíferos, los machos tienen normalmente la constitución ćromosona sexual XY (referencia L. Sachs, 1954, Ann. Engen., 18, 255), y las hembras la constitución de cromosona sexual XX. En las personas sexualmente normales, el análisis de la constitución del cromosona sexual da, pues, el diagnóstico del sexo”. Durante una entrevista personal con el doctor Leo Sachs, en su laboratorio de Rejovot, el biólogo, de treinta y un años de edad, amplió su declaración. De acuerdo con sus teorías, la genética ha investigado la transmisión de rasgos hereditarios mediante experimentación y observación. Actualmente se sabe que la materia genética la contienen, en su mayor parte, corpúsculos de tamaño microscópico 
llamados cromosonas, que se encuentran en los núcleos de las células. Acentúa luego el hecho de que los cromosonas pueden estudiarse en el microscopio, con el fin de determinar su número, forma o medios de reproducción. En el curso de estos últimos años se han desarrollado métodos por medio de los cuales puece saberse algo sobre la composición química de estos cromosomas. Este último campo de estudio es una de las mayores preocupaciones, tanto del doctor Sachs como de su colega el doctor Danon.

Ambos biólogos están haciendo investigaciones con respecto a la relación que existe entre la conducta y la estructura de los cromosomas y su influencia en la herencia. Por ejemplo, si puede relacionarse una composición química determinada con un tipo de conducta hereditaria, sería posible deducir las caracteI ísticas químicas de la herencia. Una sustancia química determinada, ácido nucleico desoxiriboso, del cual hay buenas pruebas de que es uno de los más importantes —o quizá el más importante- de los elementos químicos que constituyen la materia hereditaria, se encuentra solo en los cromosomas.

Mientras estudiaban algunas de las propiedades químicas especiales de los cromosomas sexuales, los doctores Sachs y Danon lograron hacer su descubrimiento.

\section{¿Qué es lo que determina si será niño o niña?}

Hace muchos años que se sabe que en muchas especies, incluso la de los seres humanos, el macho y la hembra tienen diferentes cromosomas sexuales. Los hombres de ciencia designan a los del macho como $\mathrm{X}$ e $\mathrm{Y}, \mathrm{y}$ a los de las hembras como $\mathrm{X}$ y $\mathrm{X}$. Al formarse el esperma, la mitad del mismo tendrá un cromosoma $X$, y la otra mitad $Y$. Cuando se produce la fertilización, si el huevo queda fertilizado por un esperma que contiene $\mathrm{Y}$, éste producirá un niño, y si el huevo queda impregnado por un esperma $X$, el resultado será una niña. Por consiguiente la combinación $\mathrm{XY}$ produce niños, y la $\mathrm{XX}$ produce niñas. Según el coctor Sachs, estos cromosomas pueden identificarse ya sea en células que están en proceso de división o tal como se descubrió primeramente en los seres humanos, mediante la labor de tres investigadores canadienses en 1953, en células que no están divididas.

Tomando células no divididas de cualquier tejido del cuerpo humano y determinando el porcentaje de células que tiene una mancha microscópica, la cual se llama cromocentro, y que 
es la que contiene el ácido nucleico desoxiriboso, se puede decir si las células proceden de un macho o de una hembra. Una hembra con cromosoma sexual $\mathrm{XX}$, forma un cromocentro con un porcentaje mucho mayor de células que un macho cuya constitución de cromosoma sexual es $\mathrm{XY}$. Se deduce que si se pueden cbtener células de un embrión antes del nacimiento, se puede cieterminar el sexo de la criatura mientras está en el útero por medio del porcentaje de células del cromocentro.

"Como se sabía que durante el desarrollo embrionario de la criatura ésta está rodeada por un líquido, el fluído amniótico, y que este fluído contiene células desprendidas de la criatura durante el desarrollo, el flúido era una evidente fuente de análisis".

"Examinamos muestras del mismo en diversos períodos del embarazo - dice el doctor Sachs - y descubrimos que contenían células apropiadas para hacer el diagnóstico del sexo. Y como se sabe que todas las células del fluído provienen de la criatura, no parece que haya la menor posibilidad de cometer error con respecto al diagnóstico".

En cuanto al origen de las células tomadas del flúido amniótico, el ginecólogo doctor Serr, declara:

"Después de la fertilización el óvulo se divide en 2 células. Éstas 2 en 4, las 4 en 8 , y así sucesivamente hasta que se forma una bola de células, en la cual se reconocen tres capas. La capa central se convertirá en el feto propiamente dicho. La próxima capa se convertirá en el amnios que envuelve el feto. La tercera formará el corion, que constituye el forro del útero, y una parte del cual ayudará a formar la placenta. Las dos últimas capas, el amnios y el corion, se fusionan en una sola, y al final del tercer mes de embarazo se forma una bolsa completamente cerrada, en la cual flota el feto en el flúido amniótico, que probablemente se forma de la membrana amniótica. Cualquier tejido que se encuentre dentro de esta bolsa es de origen fetal. La fuente de origen de las células no se conoce bien a fondo todavía. Es evidente que son de origen fetal, porque proceden del interior ce la bolsa cerrada que contiene el feto. No obstante, su origen inmediato debe ser el desprendimiento de células cutáneas, o del conducto gastrointestinal, o quizá de la propia membrana amniótica".

Al retirar el flúido amniótico se tomaron toda clase de precauciones para evitar que se contaminaran con células procedentes de la madre. 


\section{Deducciones}

El descubrimiento de los científicos israelíes ha despertado ya interés en diversas partes del mundo. Especialmente la prensa le ha dado una enorme publicidad, por cuanto da una respuesta a la curiosidad popular sobre una de las cuestiones con que se enfrentan siempre los futuros padres. Sin embargo, al igual que en el caso del descubrimiento de la penicilina, por Fleming, por ejemplo, puede desempeñar un papel mucho más importante que el que a primera vista parece sugerir.

En la actualidad, aunque se puede predecir el sexo antes ciel alumbramiento, no se puede cambiar el sexo de la criatura. Como dicen los doctores Sachs y Danon: "El sexo del feto queda fijado en el momento de la fertilización, y para elegir el sexo de la criatura sería necesario separar el contenido " $\mathrm{X}$ " del contenido "Y" del esperma, ya que el óvulo siempre tiene cromosomas X".

\section{Otros campos de estudio}

El doctor Sachs, en la época de las controversias de Lysenko, estaba en el Instituto John Innes - uno de los centros más importantes de investigación genética de inglaterra- y repitió uno de los experimentos del investigador ruso con los tomates, en vez de limitarse a discutir sobre el particular, como hicieron otros, y obtuvo resultados que refutaban las aserciones de Lysenko. Estos resultados se publicaron en "Nature", en la edición de diciembre de 1949 y en la de febrero de 1951. A pesar de que el doctor Sachs las comunicó personalmente a Glushenko, el ayudante de Lysenko, cuando visitó Inglaterra, los científicos rusos hicieron caso omiso de dichos resultados. Se convirtieron, en cambio, en una fuente importante de referencia para los biólogos de Inglaterra. De esta forma, aunque negando la influencia del ambiente pasivo sobre las características hereditarias, el doctor Leo Sachs no tiene opiniones preformadas con respecto a las posibilidades de la ciencia, pues con los medios activos del hombre se puede dirigir, cambiar o ejercer influencia sobre las mismas. Por ejemplo, la radiación se sabe que influye en los cromosomas y su constitución hereditaria.

Uno de los campos que más le atraen, en colaboración con otros, es la conducta de los cromosomas de tejidos cancerosos. Tratan de descubrir bajo qué condiciones crecen estos cromosomas, y esto puede muy bien ejercer una enorme influencia en 
los estudios de la enfermedad y ayudar a comprenderla. Otros temas de investigación en la actualidad consisten en observar las características de las marcas dactilares y los grupos sanguíneos de diversas comunidades judias, comparadas con las no judías.

Estas diversas actividades en el campo de las investigaciores de ambos biólogos se ven estimuladas por la actitud más que liberal de la institución en la cual trabajan. "El Instituto Científico Weizmann concede a sus hombres de ciencia carta blanca para que elijan su propio campo de investigación después de someter a grandes rasgos la dirección general de su búsqueda", manifiestan los doctores Sachs y Danon.

\section{Los hombres de ciencia $y$ sus instituciones}

El doctor Leo Sachs, que posee su diploma universitario de la Universidad de Cambridge, donde presentó su tesis sobre la conducta de los cromosomas, entró a formar parte del cuerpo de investigadores del Instituto Científico Weizmann, a comienzos de 1952. Dirige la Seción de Genética del Departamento Experimental de Biología a cargo del profesor Isaac Berenblum. Antes de establecerse en Israel, en el año 1951, llevó a cabo labores de irnvestigación en el Instituto de Genética de la Universidad de Lund, de Suecia.

La doctora Matilde Danon, de veintinueve años de edad, trabaja con el doctor Sachs. En la actualidad lleva ya tres años en Israel, siempre en el Instituto Weizmann. Se graduó en la Universidad de Ginebra, Suiza, y obtuvo su diploma con una tesis scbre la estructura de los cromosomas.

Al igual que el doctor Sachs, el tercer científico dedicado a esta materia, el doctor David Serr emigró a Israel desde Inglaterra en 1951, época en que entró a formar parte del Departamento de Obstetricia y Ginecología del Hospital de la Universidad Rothschild-Hadassah, a la cabeza del cual se encuentra una figura tan prestigiosa como lo es el profesor Bernard Zondeck, famoso por su análisis para la determinación del embarazo. El doctor Serr se graduó en la Universidad de Leeds y tiene treinta $\varepsilon$ ños de edad.

El Instituto Científico Weizmann situado en Rehovoth, Israel, lleva a cabo investigaciones en el dominio de la ciencia pura, pero también se dedica a ensayos en el terreno práctico. Dentro de su radio de acción figuran por lo menos 60 proyectos, cistribuídos entre unos 12 departamentos y secciones. 
El Hospital de la Universidad Rothschild-Hadassah es la institución que han formado en combinación la Organización Médica Hadassah de Israel - una de las más grandes del país y sostenida por fondos de los Estados Unidos- y la Universidad Hebrea de Jerusalén. Sirve a la Universidad como parte de su Facultad de Medicina e investigaciones médicas y a Hadassah como centro para el tratamiento y la investigación en las técnicas médicas más adelantadas de la medicina moderna.

para ecuanimidad....
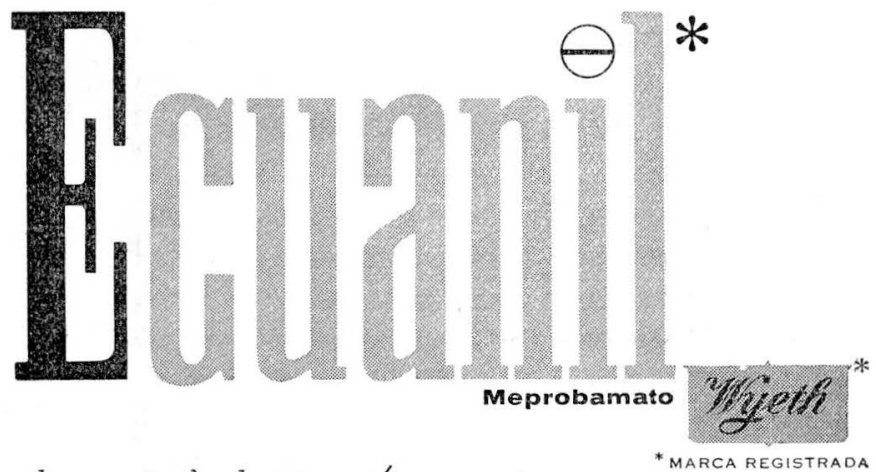

Estados de ansiedad y tensión nerviosa.

Estados neurológicos por espasmo muscular.

Ciertos trastornos convulsivos. 